\section{Hohes Thromboembolie-Risiko bei Rheumapatienten}

Bei Patienten mit rheumatoider Arthritis (RA) tritt zwei- bis dreifach häufiger eine venöse Thromboembolie (VTE) auf als in der Allgemeinbevölkerung. Das ergab die Analyse eines großen nationalen Registers der Universität Taichung in Taiwan. Ausgewertet wurden knapp 30000 RA-Patienten, die zunächst noch keine tiefe Venenthrombose (TVT) und keine Lungenembolie hatten. Als Vergleichsgruppe dienten 117000 Personen ohne RA. Pro 10000 Personenjahre traten bei den RA-Patienten etwa 11 TVT auf, in der Kontrollgruppe nur 3,2. Die TVT-Rate war damit bei Patienten mit RA unter Berücksichtigung weiterer Risikofaktoren 3,3-fach erhöht. Doppelt so häufig wie in der Kontrollgruppe kam es bei RA-Patienten zu Lungenembolien (3,6 vs. 1,75 Ereignisse pro 10000 Personenjahre).

- Ann Rheum Dis 2013, online first: 7.8.2013; doi:10.1136/annrheumdis-2013-203380

Reinfektion aus der Socke

\section{Ein 40-Grad-Waschgang lässt den Fußpilz kalt}

Wer sich wundert, weshalb er trotz regelmäßigen Sockenwechsels seinen Fußpilz nicht los wird, der sollte sein Waschverhalten überprüfen. Israelische Dermatologen unterzo-

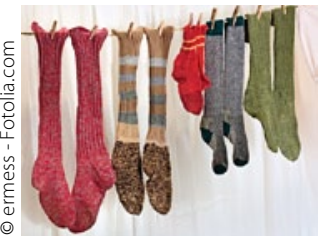

Ferse mit Trichophyton (T.) rubrum und Aspergillus spp. kontaminiert. Bei 29 Proben ergab sich auch nach der 40-Grad-Wäsche in der Kultur noch ein Pilzwachstum. Höhere Waschtemperaturen gen die Socken von Patienten mit Onychomykose oder Tinea pedis nach sechsstündigem Tragen Waschgängen bei verschiedenen Temperaturen.

66 bzw. 72 der 81 ungewaschenen Sockenproben waren im Zehenbereich bzw. an der vertrugen die Pilze deutlich schlechter. Nur $6 \%$ der Socken waren auch nach der 60-Grad-Wäsche noch kontaminiert.

- International Journal of Dermatology 2013; online: 24 JUL 2013; doi: 10.1111/ijd.12167

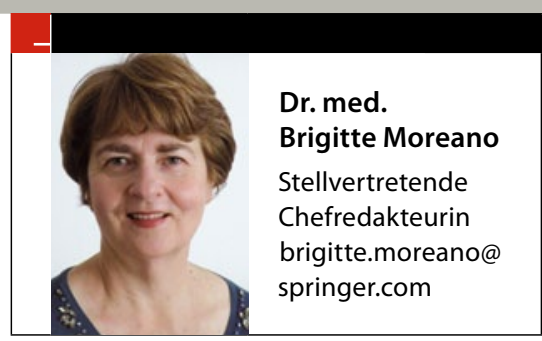

Körpergröße als Indikator

\title{
Das Krebsrisiko mit dem Zollstock messen
}

Bei Frauen wächst mit der Körpergröße die Krebsgefahr. In der Women's Health Initiative (WHI) waren 144701 postmenopausale Frauen im Median zwölf Jahre lang verfolgt worden. In dieser Zeit wurden 20928 Krebserkrankungen diagnostiziert. Krebsrate und Körpergröße waren positiv und signifikant miteinander assoziiert. Wurden etablierte Einflussfaktoren wie Alter, BMI, Rauchen, Alkoholkonsum, Hormontherapie etc. berücksichtigt, stieg pro $10 \mathrm{~cm}$ Körpergröße das Krebsrisiko um 13\%. Die Risikosteigerung pro $10 \mathrm{~cm}$ bewegte sich zwischen $29 \%$ (Schilddrüsenkarzinom, Multiples Myelom) und $13 \%$ (Brustkrebs). Für maligne Hirn- und Lungentumoren sowie für Non-HodgkinLymphome war die Korrelation mit der Körpergröße grenzwertig signifikant.

Kognitive Leistung bei Älteren

\section{Schwankender Blutdruck geht auf den Geist}

Je stärker die Blutdruckwerte von älteren Patienten schwanken, desto schlechter schneiden sie in kognitiven Tests ab. In der PROSPER-(Prospective Study of Pravastatin in the Elderly at Risk-)Studie wurde bei 5461 über 70-jährigen Patienten über rund drei Jahre hinweg alle drei Monate der Blutdruck gemessen. Am Studienende erfolgten ausführliche kognitive Tests. Ein Vergleich der Probanden mit hoher Blutdruckvariabilität mit jenen, deren Blutdruck nur wenig schwankte, zeigte: Schwankender Druck geht mit schlechterer kognitiver Funktion einher, und zwar unabhängig vom Durchschnittswert des Blutdrucks. Überdies fanden sich bei Teilnehmern mit hohen Druckschwankungen in der Magnetresonanztomografie häufiger kortikale Infarkte und ein niedrigeres Hippocampusvolumen.

- BMJ 2013; 347: f4600; doi: 10.1136/bmj.f4600
- Cancer Epidemiol Biomarkers Prev 2013, Online 25. Juli; doi: 10.1158/1055-9965.EPI-13-0305

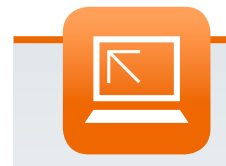

Top gelesen auf springermedizin.de

Blickdiagnose interaktiv „Rot und wund am Labiengrund" -4563968

Vorhofflimmern - da kommt was auf uns zu! \& 4596554 Klagen gegen Allgemeinärzte: Was sind die häufigsten Gründe? > 4574246

- Diese Artikel finden Sie, indem Sie die ID-Nummern in die Suche eingeben. 